\title{
Global solutions to a class of nonlinear damped wave operator equations
}

\author{
Zhigang $\mathrm{Pan}^{1^{*}}$, Zhilin $\mathrm{Pu}^{2}$ and Tian $\mathrm{Ma}^{1}$
}

\author{
* Correspondence: pzg555@yeah. \\ net \\ ${ }^{1}$ Yangtze Center of Mathematics, \\ Sichuan University, Chengdu \\ 610064, P. R. China \\ Full list of author information is \\ available at the end of the article
}

\section{Abstract \\ This study investigates the existence of global solutions to a class of nonlinear damped wave operator equations. Dividing the differential operator into two parts, variational and non-variational structure, we obtain the existence, uniformly bounded and regularity of solutions. \\ Mathematics Subject Classification 2000: 35L05; 35A01; 35L35. \\ Keywords: nonlinear damped wave operator equations, global solutions, uniformly bounded, regularity}

\section{Introduction}

In recent years, there have been extensive studies on well-posedness of the following nonlinear variational wave equation with general data:

$$
\begin{cases}\partial_{t}{ }^{2} u-c(u) \partial_{x}\left(c(u) \partial_{x} u\right)=0 & \text { in }(0, \infty) \times \mathbf{R}, \\ \left.u\right|_{t=0}=u_{0} & \text { on } \mathbf{R}, \\ \left.\partial_{t} u\right|_{t=0}=u_{1} & \text { on } \mathbf{R},\end{cases}
$$

where $c(\cdot)$ is given smooth, bounded, and positive function with $c^{\prime}(\cdot) \geq 0$ and $c^{\prime}\left(u_{0}\right)>$ $0, u_{0} \in H^{1}(\mathbf{R}), u_{1}(x) \in L^{2}(\mathbf{R})$. Equation (1.1) appears naturally in the study for liquid crystals [1-4]. In addition, Chang et al. [5], Su [6] and Kian [7] discussed globally Lipschitz continuous solutions to a class one dimension quasilinear wave equations

$$
\left\{\begin{array}{l}
u_{t t}-\left(p\left(\rho(x), u_{x}\right)\right)_{x}=\rho(x) h\left(\rho(x), u, u_{x}\right) \\
u(x, 0)=u_{0}(x) \\
u_{t}(x, 0)=\omega_{0}(x)
\end{array}\right.
$$

where $(x, t) \in \mathbf{R} \times \mathbf{R}^{+}, u_{0}(x), \omega_{0}(x) \in \mathbf{R}$. Furthermore, Nishihara [8] and Hayashi [9] obtained the global solution to one dimension semilinear damped wave equation

$$
\left\{\begin{array}{l}
u_{t t}+u_{t}-u_{x x}=f(u),(t, x) \in \mathbf{R}^{+} \times \mathbf{R}^{+} \\
\left(u, u_{t}\right)(0, x)=\left(u_{0}, u_{1}\right)(x)
\end{array}\right.
$$

Ikehata [10] and Vitillaro [11] proved global existence of solutions for semilinear damped wave equations in $\mathbf{R}^{N}$ with noncompactly supported initial data or in the energy space, in where the nonlinear term $f(u)=|u|^{p}$ or $f(u)=0$ is too special; some authors [12-14] discussed the regularity of invariant sets in semilinear wave equation, but they didn't refer to any the initial value condition of it. Unfortunately, it is difficulty to classify a class wave operator equations, since the differential operator 
structure is too complex to identify whether have variational property. Our aim is to classify a class of nonlinear damped wave operator equations in order to research them more extensively and go beyond the results of [12].

In this article, we are interested in the existence of global solutions of the following nonlinear damped wave operator equations:

$$
\left\{\begin{array}{l}
\frac{d^{2} u}{d t^{2}}+k \frac{d u}{d t}=G(u), k>0 \\
u(x, 0)=\varphi(x) \\
u_{t}(x, 0)=\psi(x)
\end{array}\right.
$$

where $G: X_{2} \times \mathbf{R}^{+} \rightarrow X_{1}^{*}$ is a mapping, $X_{2} \subset X_{1}, X_{1}, X_{2}$ are Banach spaces and $X_{1}^{*}$ is the dual spaces of $X_{1}, \mathbf{R}^{+}=[0, \infty), u=u(x, t)$. If $k>0,(1.4)$ is called damped wave equation. We obtain the existence, uniformly bounded and regularity of solutions by dividing the differential operator $G(u)$ into two parts, variational and non-variational structure.

\section{Preliminaries}

First we introduce a sequence of function spaces:

$$
\left\{\begin{array}{l}
X \subset H_{2} \subset X_{2} \subset X_{1} \subset H_{1} \\
X_{2} \subset H_{1} \subset H_{1}
\end{array}\right.
$$

where $H, H_{1}, H_{2}$ are Hilbert spaces, $X$ is a linear space, $X_{1}, X_{2}$ are Banach spaces and all inclusions are dense embeddings. Suppose that

$$
\left\{\begin{array}{l}
L: X \rightarrow X_{1} \text { is one to one dense linear operator, } \\
\langle L u, v\rangle_{H}=\langle u, v\rangle_{H}, \quad \forall u, v \in X .
\end{array}\right.
$$

In addition, the operator $L$ has an eigenvalue sequence

$$
L e_{k}=\lambda_{k} e_{k}, \quad(k=1,2, \ldots)
$$

such that $\left\{e_{k}\right\} \subset X$ is the common orthogonal basis of $H$ and $H_{2}$. We investigate the existence of global solutions of the Equation (1.4), so we need define its solution. Firstly, in Banach space $X$, introduce

$$
L^{p}((0, T), X)=\left\{u:(0, T) \rightarrow X \mid \int_{0}^{T}\|u\|^{p} d t<\infty\right\},
$$

where $p=\left(p_{1}, p_{2}, \ldots, p_{m}\right), p_{i} \geq 1(1 \leq i \leq m)$,

$$
\|u\|^{p}=\sum_{k=1}^{m}|u|_{k}^{p_{k}}
$$

where $|\cdot|_{k}$ is semi-norm in $X$, and $\|\cdot\|_{X}=\sum_{i=1}^{m}|\cdot|_{i}$. Similarily, we can define

$$
W^{1, p}((0, T), X)=\left\{u:(0, T) \rightarrow X \mid u, \quad u^{\prime} \in L^{p}((0, T), X)\right\} .
$$

Let $L_{\mathrm{loc}}^{p}((0, \infty), X)=\left\{u(t) \in X \mid u \in L^{p}((0, T), X), \forall T>0\right\}$.

Definition 2.1. Set $(\phi, \psi) \in X_{2} \times H_{1}, u \in W_{\text {loc }}^{1, \infty}\left((0, \infty), H_{1}\right) \bigcap L_{\mathrm{loc}}^{\infty}\left((0, \infty), X_{2}\right)$ is called a globally weak solution of (1.4), if for $\forall v \in X_{1}$, it has 


$$
\left\langle u_{t}, v\right\rangle_{H}+k\langle u, v\rangle_{H}=\int_{0}^{t}\langle G u, v\rangle d t+k\langle\varphi, v\rangle_{H}+\langle\psi, v\rangle_{H} .
$$

Definition 2.2. Let $Y_{1}, Y_{2}$ be Banach spaces, the solution $u(t, \phi, \psi)$ of (1.4) is called uniformly bounded in $Y_{1} \times Y_{2}$, if for any bounded domain $\Omega_{1} \times \Omega_{2} \subset Y_{1} \times Y_{2}$, there exists a constant $C$ which only depends the domain $\Omega_{1} \times \Omega_{2}$, such that

$$
\|u\|_{Y_{1}}+\left\|u_{t}\right\|_{Y_{2}} \leq C, \quad \forall(\varphi, \psi) \in \Omega_{1} \times \Omega_{2} \text { and } t \geq 0 .
$$

Definition 2.3. A mapping $G: X_{2} \rightarrow X_{1}^{*}$ is called weakly continuous, if for any sequence $\left\{u_{n}\right\} \subset X_{2}, u_{n} \rightarrow u_{0}$ in $X_{2}$,

$$
\lim _{n \rightarrow \infty}\left\langle G\left(u_{n}\right), v\right\rangle=\left\langle G\left(u_{0}\right), v\right\rangle, \quad \forall v \in X_{1} .
$$

Lemma 2.1. [15]Let $H_{2}, H$ be Hilbert spaces, and $H_{2} \subset H$ be a continuous embedding. Then there exists a orthonormal basis $\left\{e_{k}\right\}$ of $H$, and also is one orthogonal basis of $\mathrm{H}_{2}$.

Proof. Let $I: H_{2} \rightarrow H$ be imbedded. According to assume $I$ is a linear compact operator, we define the mapping $A: H_{2} \rightarrow H$ as follows

$$
\langle A u, v\rangle_{H_{2}}=\langle I u, v\rangle_{H}=\langle u, v\rangle_{H}, \quad \forall v \in H_{2} .
$$

obviously, $\mathrm{A}: \mathrm{H}_{2} \rightarrow \mathrm{H}_{2}$ is linear symmetrical compact operator and positive definite. Therefore, $A$ has a complete eigenvalue sequence $\left\{\lambda_{k}\right\}$ and eigenvector sequence $\left\{\tilde{e}_{k}\right\} \subset H_{2}$ such that

$$
A \tilde{e}_{k}=\lambda_{k} \tilde{e}_{k}, \quad k=1,2, \ldots
$$

and $\left\{\tilde{e}_{k}\right\}$ is orthogonal basis of $H_{2}$. Hence

$$
\left\langle\tilde{e}_{i}, \tilde{e}_{j}\right\rangle_{H}=\left\langle A \tilde{e}_{i}, \tilde{e}_{j}\right\rangle_{H_{2}}=\lambda_{i}\left|\tilde{e}_{i}, \tilde{e}_{j}\right|_{H_{2}}=0, \quad \text { if } i \neq j
$$

it implies $\left\{\tilde{e}_{i}\right\}$ is also orthogonal sequence of $H$. Since $H_{2} \subset H$ is dense, $\left\{\tilde{e}_{i}\right\}$ is also orthogonal sequence of $H$, so $\left\{e_{i}\right\}=\left\{\tilde{e}_{i} /\left\|\tilde{e}_{i}\right\|_{H}\right\}$ is norm orthogonal basis of $H$. The proof is completed.

Now, we introduce an important inequality

Lemma 2.2. [16] (Gronwall inequality) Let $x(t), y(t), z(t)$ be real function on $[a, b]$, where $x(t) \geq 0, \forall a \leq t \leq b, z(t) \in C[a, b], y(t)$ is differentiable on $[a, b]$. If the inequality as follows is hold

$$
z(t) \leq \gamma(t)+\int_{a}^{t} x(\tau) z(\tau) d \tau, \quad a \leq t \leq b,
$$

then

$$
z(t) \leq y(a) e^{\int_{a}^{t} x(s) d s}+\int_{a}^{t} e^{\int_{a}^{t} x(\tau)} \frac{d y}{d s} d s .
$$




\section{Main results}

Suppose that $G=A+B: X_{2} \times \mathbf{R}^{+} \rightarrow X_{1}^{*}$. Throughout of this article, we assume that

(i) There exists a function $F \in C^{1}: X_{2} \rightarrow \mathbf{R}^{1}$ such that

$$
\langle A u, L v\rangle=\langle-D F(u), v\rangle, \quad \forall u, v \in X
$$

(ii) Function $F$ is coercive, if

$$
F(u) \rightarrow \infty \Leftrightarrow\|u\|_{X_{2}} \rightarrow \infty
$$

(iii) $B$ as follows

$$
|\langle B u, L v\rangle| \leq C_{1} F(u)+C_{2}\|v\|_{H_{1}}^{2}, \quad \forall u, v \in X
$$

for some $g \in L_{\text {loc }}^{1}(0, \infty)$.

Theorem 3.1. Set $G: X_{2} \times \mathbf{R}^{+} \rightarrow X_{1}^{*}$ is weakly continuous, $(\phi, \psi) \in X_{2} \times H_{1}$, then we obtain the results as follows:

(1) If $G=A$ satisfies the assumption (i) and (ii), then there exists a globally weak solution of (1.4)

$$
u \in W_{\mathrm{loc}}^{1, \infty}\left((0, \infty), H_{1} \bigcap L_{\mathrm{loc}}^{\infty}\left((0, \infty), X_{2}\right)\right)
$$

and $u$ is uniformly bounded in $X_{2} \times H_{1}$;

(2) If $G=A+B$ satisfies the assumption (i), (ii) and (iii), then there exists a globally weak solution of (1.4)

$$
u \in W_{\mathrm{loc}}^{1, \infty}\left((0, \infty), H_{1}\right) \bigcap L_{\mathrm{loc}}^{\infty}\left((0, \infty), X_{2}\right) ;
$$

(3) Furthermore, if $G=A+B$ satisfies

$$
|\langle G u, v\rangle| \leq \frac{1}{2}\|v\|_{H}^{2}+C F(u)+g(t)
$$

for some $g \in L_{\mathrm{loc}}^{1}(0, \infty)$, then $u \in W_{\mathrm{loc}}^{2,2}((0, \infty), H)$.

Proof. Let $\left\{e_{k}\right\} \subset X$ be the public orthogonal basis of $H$ and $H_{2}$, satisfies (2.3).

Note

$$
\left\{\begin{array}{l}
X_{n}=\left\{\sum_{i=1}^{n} \alpha_{i} e_{i} \mid \alpha_{i} \in \mathbf{R}^{1}\right\}, \\
\widetilde{X}_{n}=\left\{\sum_{j=1}^{n} \beta_{j}(t) e_{j} \mid \beta_{j} \in C^{2}[0, \infty)\right\} .
\end{array}\right.
$$


From the assumption, we know $L X_{n}=X_{n}, L \widetilde{X}_{n}=\widetilde{X}_{n}$, apply the Galerkin method to make truncate in $\widetilde{X}_{n}$ :

$$
\left\{\begin{array}{l}
\frac{d^{2} u_{i}}{d t^{2}}+k \frac{d u_{i}}{d t}=\left\langle G\left(u_{n}\right), e_{i}\right\rangle, 1 \leq i \leq n \\
u_{i}(x, 0)=\left\langle\varphi, \quad e_{i}\right\rangle_{H^{\prime}} \\
u^{\prime}{ }_{i}(x, 0)=\left\langle\psi, e_{i}\right\rangle_{H}
\end{array}\right.
$$

there exists $u_{n}=\sum_{i=1}^{n} u_{i}(t) e_{i} \in C^{2}\left((0, \infty), X_{n}\right)$ for any $v \in \widetilde{X}_{n}$ satisfies

$$
\int_{0}^{t}\left\langle\frac{d^{2} u_{n}}{d t^{2}}+k \frac{d u_{n}}{d t}, v\right\rangle_{H} d t=\int_{0}^{t}\left\langle G u_{n}, v\right\rangle d t
$$

for any $v \in X_{n}$, it yields that

$$
\left\langle\frac{d u_{n}}{d t}, v\right\rangle_{H}+k\left\langle u_{n}, v\right\rangle_{H}=\int_{0}^{t}\left\langle G u_{n}, v\right\rangle d t+k\langle\varphi, v\rangle_{H}+\langle\psi, v\rangle_{H}
$$

(1) If $G=A, u_{n} \in \widetilde{X}_{n}$ substitute $v=\frac{d}{d t} L u_{n}$ into (3.7), we get

$$
\int_{0}^{t}\left\langle\frac{d^{2} u_{n}}{d t^{2}}+k \frac{d u_{n}}{d t}, \frac{d}{d t} L u_{n}\right\rangle_{H_{1}} d t=\int_{0}^{t}\left\langle G u_{n}, \frac{d}{d t} L u_{n}\right\rangle d t
$$

combine condition (2.2) with (3.1), we get

$$
\begin{gathered}
\int_{0}^{t} \int_{\Omega} \frac{d^{2} u_{n}}{d t^{2}} \frac{d u_{n}}{d t} d x d t+\int_{0}^{t} \int_{\Omega} k \frac{d u_{n}}{d t} \frac{d u_{n}}{d t} d x d t+\int_{0}^{t} D F\left(u_{n}\right) \frac{d u_{n}}{d t} d x d t=0 \\
\int_{0}^{t} \frac{1}{2} \frac{d}{d t}\left\|\frac{d u_{n}}{d t}\right\|_{H_{1}}^{2} d t+k \int_{0}^{t}\left\|\frac{d u_{n}}{d t}\right\|_{H_{1}}^{2} d t+\int_{0}^{t} \frac{d}{d t} F\left(u_{n}\right) d t=0 \\
\frac{1}{2}\left\|\frac{d u_{n}}{d t}\right\|_{H_{1}}^{2}-\frac{1}{2}\left\|\psi_{n}\right\|_{H_{1}}^{2}+k \int_{0}^{t}\left\|\frac{d u_{n}}{d t}\right\|_{H_{1}}^{2} d t+F\left(u_{n}\right)-F\left(\varphi_{n}\right)=0
\end{gathered}
$$

consequently, we get

$$
F\left(u_{n}\right)+\frac{1}{2}\left\|u_{n}^{\prime}\right\|_{H_{1}}^{2}+k \int_{0}^{t}\left\|u_{n}^{\prime}\right\|_{H_{1}}^{2} d t=F\left(u_{n}\right)+\frac{1}{2}\left\|\psi_{n}\right\|_{H_{1}}^{2} .
$$

Assume $\phi \in H_{2}$, combine(2.2)with(2.3), we know $\left\{e_{n}\right\}$ is also the orthogonal basis of $H_{1}$, then $\phi_{n} \rightarrow \phi$ in $H_{2}, \psi_{n} \rightarrow \psi$ in $H_{1}$, owing to $H_{2} \subset X_{2}$ is embedded, so

$$
\left\{\begin{array}{l}
\varphi_{n} \rightarrow \varphi \text { in } X_{2} \\
\psi_{n} \rightarrow \psi \text { in } X_{1}
\end{array}\right.
$$

due to the condition (3.6), from (3.9)and (3.10) we easily know

$$
\left\{u_{n}\right\} \subset W_{\text {loc }}^{1, \infty}\left((0, \infty), H_{1}\right) \bigcap L_{\text {loc }}^{\infty}\left((0, \infty), X_{2}\right) \text { is bounded }
$$


consequently, assume that

$$
u_{n} \rightarrow u_{0} \text { in } W_{\mathrm{loc}}^{1, \infty}\left((0, \infty), H_{1}\right) \bigcap L_{\mathrm{loc}}^{\infty}\left((0, \infty), X_{2}\right) \text { a.e. } t>0
$$

i.e. $u_{n} \rightarrow u_{0}$ in $X_{2}$ a.e. $t>0$, and $G$ is weakly continuous, so

$$
\lim _{n \rightarrow \infty}\left\langle G u_{n}, v\right\rangle=\left\langle G u_{0}, v\right\rangle .
$$

By (3.8), we have

$$
\begin{gathered}
\lim _{n \rightarrow \infty}\left[\left\langle\frac{d u_{n}}{d t}\right\rangle_{H}+k\left\langle u_{n}, v\right\rangle_{H}\right]=\lim _{n \rightarrow \infty} \int_{0}^{t}\left\langle G u_{n}, v\right\rangle d t+k\langle\varphi, v\rangle_{H}+\langle\psi, v\rangle_{H} \\
\left\langle\frac{d u_{0}}{d t}, v\right\rangle_{H}+k\left\langle u_{0}, v\right\rangle_{H}=\int_{0}^{t}\left\langle G u_{0}, v\right\rangle d t+k\langle\varphi, v\rangle_{H}+\langle\psi, v\rangle_{H}
\end{gathered}
$$

it indicates for any $v \in \bigcup_{n=1}^{\infty} X_{n} \subset X_{2}$, it holds. Hence, for any $v \in X_{2}$, we have

$$
\left\langle\frac{d u_{0}}{d t}, v\right\rangle_{H}+k\left\langle u_{0}, v\right\rangle_{H}=\int_{0}^{t}\left\langle G u_{0}, v\right\rangle d t+k\langle\varphi, v\rangle_{H}+\langle\psi, v\rangle_{H}
$$

Consequently, $u_{0}$ is a globally weak solution of (1.4).

Furthermore, by (3.9) and (3.10), for any $R>0$, there exists a constant $C$ such that if

$$
\|\varphi\|_{X_{2}}+\|\psi\|_{H_{1}} \leq R
$$

then the weak solution $u(t, \phi, \psi)$ of (1.4) satisfies

$$
\|u(t, \varphi, \psi)\|_{X_{2}}+\left\|u_{t}(t, \varphi, \psi)\right\|_{H_{1}} \leq C . \quad \forall t \geq 0
$$

Assume $(\phi, \psi) \in X_{2} \times H_{1}$ satisfies (3.12), by $H_{2} \subset X_{2}$ is dense. May fix $\phi_{n} \in H_{2}$ such that

$$
\left\|\varphi_{n}\right\|_{X_{2}}+\|\psi\|_{H_{1}} \leq R, \quad \lim _{n \rightarrow \infty} \varphi_{n}=\varphi \text { in } X_{2}
$$

by (3.13), the solution $\left\{u\left(t, \quad \phi_{n}, \psi\right)\right\}$ of (1.4) is bounded in $W_{\text {loc }}^{1, \infty}\left((0, \infty), H_{1}\right) \cap L_{\text {loc }}^{\infty}\left((0, \infty), X_{2}\right)$ a.e. $t>0$.

Therefore, assume $u\left(t, \phi_{n}, \psi\right) \rightarrow u$ in $W_{\text {loc }}^{1, \infty}\left((0, \infty), H_{1}\right) \bigcap L_{\text {loc }}^{\infty}\left((0, \infty), X_{2}\right)$ then $u(t)$ is a weak solution of (1.4), it satisfies uniformly bounded of (3.13). So the conclusion (1) is proved.

(2) If $G=A+B, u_{n} \in \tilde{X}_{n}$, substitute $v=\frac{d}{d t} L u_{n}$ into (3.7), we get

$$
\begin{aligned}
& \int_{0}^{t}\left[\left\langle\frac{d^{2} u_{n}}{d t^{2}}, \frac{d}{d t} L u_{n}\right\rangle_{H_{1}}\right]+k\left\langle\frac{d u_{n}}{d t}, \frac{d}{d t} L u_{n 1}\right\rangle_{H_{1}} d t \\
= & \int_{0}^{t}\left[\left\langle A u_{n}, \frac{d u_{n}}{d t}\right\rangle+\left\langle B u_{n}, \frac{d u_{n}}{d t}\right\rangle\right] d t
\end{aligned}
$$


combine the condition (2.2) and (3.1), we have

$$
\begin{aligned}
& \int_{0}^{t} \int_{\Omega} \frac{d^{2} u_{n}}{d t^{2}} \frac{d u_{n}}{d t} d x d t+k \int_{0}^{t} \int_{\Omega} \frac{d u_{n}}{d t^{2}} \frac{d u_{n}}{d t} d x d t+\int_{0}^{t}\left\langle D F\left(u_{n}\right) \frac{d u_{n}}{d t}\right\rangle d t \\
= & \int_{0}^{t}\left\langle B u_{n}, \frac{d u_{n}}{d t}\right\rangle d t \\
& \int_{0}^{t} \frac{1}{2} \frac{d}{d t}\left\|\frac{d u_{n}}{d t}\right\|_{H_{1}}^{2} d t+k \int_{0}^{t}\left\|\frac{d u_{n}}{d t}\right\|_{H_{1}}^{2} d t+\int_{0}^{t} \frac{d}{d t} F\left(u_{n}\right) d t \\
= & \int_{0}^{t}\left\langle B u_{n}, \frac{d u_{n}}{d t}\right\rangle d t \\
& \frac{1}{2}\left\|u_{n}^{\prime}\right\|_{H_{1}^{2}}-\frac{1}{2}\left\|\psi_{n}\right\|_{H_{1}}^{2}+k \int_{0}^{t}\left\|u_{n}^{\prime}\right\|_{H_{1}}^{2} d t+F\left(u_{n}\right)+F\left(\varphi_{n}\right) \\
= & \int_{0}^{t}\left\langle B u_{n}, \frac{d u_{n}}{d t}\right\rangle d t
\end{aligned}
$$

consequently, we have

$$
F\left(u_{n}\right)+\frac{1}{2}\left\|u_{n}^{\prime}\right\|_{H_{1}}^{2}+k \int_{0}^{t}\left\|u_{n}^{\prime}\right\|_{H_{1}}^{2} d t=\int_{0}^{t}\left\langle B u_{n}, \frac{d u_{n}}{d t}\right\rangle d t+F\left(\varphi_{n}\right)+\frac{1}{2}\left\|\psi_{n}\right\|_{H_{1}}^{2}
$$

by the condition (3.3),(3.14)implies

$$
F\left(u_{n}\right)+\frac{1}{2}\left\|u_{n}^{\prime}\right\|_{H_{1}}^{2} \leq C \int_{0}^{t}\left[F\left(u_{n}\right)+\frac{1}{2}\left\|u_{n}^{\prime}\right\|_{H_{1}}^{2}\right] d t+f(t)
$$

where $f(t)=\int_{0}^{t} g(\tau) d t+\frac{1}{2}\|\psi\|_{H_{1}}^{2}+\sup _{n} F\left(\varphi_{n}\right)$.

by Gronwall inequality [Lemma(2.2)], from (3.15) we easily know:

$$
F\left(u_{n}\right)+\frac{1}{2}\left\|u_{n}^{\prime}\right\|_{H_{1}}^{2} \leq f(0) e^{C^{t}}+\int_{0}^{t} f^{\prime}(\tau) e^{C(t-\tau)} d \tau
$$

it implies that, for any $0<T<\infty$

$$
\left\{u_{n}\right\} \subset W^{1, \infty}\left((0, T), X_{2}\right) \bigcap L^{\infty}\left((0, T), X_{2}\right) \text { is bounded. }
$$

now, use the same way as (1), we can obtain the result (2).

(3) If the condition (3.4) is hold, $u_{n} \in \widetilde{X}_{n}$, substitute $v=\frac{d^{2} u}{d t^{2}}$ into (3.7), we can get

$$
\int_{0}^{t}\left[\left\langle\frac{d^{2} u_{n}}{d t^{2}}, \frac{d^{2} u_{n}}{d t^{2}}\right\rangle_{H}+k\left\langle\frac{d u_{n}}{d t}, \frac{d^{2} u_{n}}{d t^{2}}\right\rangle_{H}\right] d t=\int_{0}^{t}\left\langle G u_{n}, \frac{d^{2} u_{n}}{d t^{2}}\right\rangle d t
$$


then

$$
\begin{aligned}
& \int_{0}^{t}\left\langle\frac{d^{2} u_{n}}{d t^{2}}, \frac{d^{2} u_{n}}{d t^{2}}\right\rangle_{H} d t+\frac{k}{2} \int_{0}^{t} \frac{d}{d t}\left\|u_{n}^{\prime}(t)\right\|_{H}^{2} d t \\
\leq & \int_{0}^{t}\left[\frac{1}{2}\left\|u^{\prime \prime}{ }_{n}(t)\right\|_{H}^{2}+C F\left(u_{n}\right)+g(t)\right] d t \\
& \int_{0}^{t}\left\langle\frac{d^{2} u_{n}}{d t^{2}}, \frac{d^{2} u_{n}}{d t^{2}}\right\rangle_{H} d t+\frac{k}{2}\left\|u_{n}^{\prime}\right\|_{H}^{2} \\
\leq & \frac{k}{2}\left\|\psi_{n}\right\|_{H}^{2}+\int_{0}^{t}\left[\frac{1}{2}\left\|\frac{d^{2} u_{n}}{d t^{2}}\right\|_{H}^{2}+C F\left(u_{n}\right)+g(\tau)\right] d \tau
\end{aligned}
$$

by (3.16), it implies that

$$
\int_{0} t\left\|\frac{d^{2} u_{n}}{d t^{2}}\right\|_{H}^{2} d \tau \leq C, \quad(C>0)
$$

consequently, for any $0<T<\infty$

$$
\left\{u_{n}\right\} \subset W^{2,2}((0, T), H) \text { is bounded. }
$$

it implies that $u \in W^{2,2}((0, T), H)$, the main theorem (3.1) has been proved.

\section{Acknowledgements}

The author is very grateful to the anonymous referees whose careful reading of the manuscript and valuable comments enhanced presentation of the manuscript. Foundation item: the National Natural Science Foundation of China (No. 10971148).

\section{Author details}

${ }^{1}$ Yangtze Center of Mathematics, Sichuan University, Chengdu 610064, P. R. China ${ }^{2}$ College of Mathematics and Software Science, Sichuan Normal University, Chengdu, Sichuan 610066, P. R. China

\section{Authors' contributions}

All authors typed, read and approved the final manuscript.

\section{Competing interests}

The authors declare that they have no competing interests.

Received: 6 October 2011 Accepted: 13 April 2012 Published: 13 April 2012

\section{References}

1. Glassey, RT, Hunter, JK, Zheng, Y: Singularities and oscillations in a nonlinear variational wave equations. In IMA, vol. 91, Springer, New York (1997)

2. Hunter, JK, Zheng, Y: On a nonlinear hyperbolic variational equation I and II. Arch Rationl Mech Anal 129, 305-353 (1995). 355-383

3. Hunter, JK, Staxton, RA: Dynamics of director fields. SIAM J Appl Math Phys. 51, 1498-1521 (1991)

4. Saxton, RA: Dynamic instability of the liquid crystal director. In: Lindquist WB (ed.) Current Progress in Hyperbolic, vol. 100, pp. 325-330. Contemporary Mathematics, AMS, Providence (1989)

5. Chang, Y, Hong, JM, Hsu, CH: Globally Lipschitz continuous solutions to a class of quasilinear wave equations. J Diff Eqn. 236, 504-531 (2007)

6. Su, YC: Global entropy solutions to a class of quasi-linear wave equations with large time-oscillating sources. J Diff Equ. 250, 3668-3700 (2011)

7. Kian, Y: Cauchy problem for semilinear wave equation with time-dependent metrics. Nonlinear Anal. 73, 2204-2212 (2010)

8. Nishihara, KJ, Zhao, HJ: Existence and nonexistence of time-global solutions to damped wave equation on half-time. Nonlinear Anal. 61, 931-960 (2005)

9. Hayashi, N, Kaikina, El, Naumkin, PI: Damped wave equation in the subcritional case. J Diff Equ. 207, 161-194 (2004)

10. Ikehata, R, Tanizawa, K: Global existence of solutions for semilinear damped wave equations in $R^{N}$ with noncompactly supported initial data. J Diff Eqn. 61, 1189-1208 (2005) 
11. Vitillaro, E: Global existence for the wave equation with nonlinear boundary damping and source terms. J Diff Equ. 186, 259-298 (2002)

12. Prizzi, M: Regularity of invarant sets in semilinear damped wave equations. J Math Anal Appl. 247, 3315-3337 (2009)

13. Rybakowski, KP: Conley index continuation for singularly perturbed hyperbolic equations. Topol Methods Nonlinear Anal. 22, 203-244 (2003)

14. Zelik, S: Asymptotic regularity of solutions of a nonautonomous damped wave equation with a critical growth exponent. Commun Pure Appl Anal. 3, 921-934 (2004)

15. Ma, T: Theorey and Method of Partial Differential Equations. Science Press, Beijing (2011)

16. Birkhoff, G, Rota, GC: Ordinary Differential Equations. pp. 72-90. John Wiley, New York (1978)

doi:10.1186/1687-2770-2012-42

Cite this article as: Pan et al:: Global solutions to a class of nonlinear damped wave operator equations. Boundary Value Problems 2012 2012:42.

\section{Submit your manuscript to a SpringerOpen ${ }^{\odot}$} journal and benefit from:

- Convenient online submission

- Rigorous peer review

- Immediate publication on acceptance

- Open access: articles freely available online

- High visibility within the field

- Retaining the copyright to your article

Submit your next manuscript at $\gg$ springeropen.com 